\title{
Uptake of Long Protein-Polyelectrolyte Nanotubes by Dendritic Cells
}

\author{
Diana G. Ramírez-Wong, ${ }^{\dagger,+}$ Saghi Saghazadeh, ${ }^{\dagger,}{ }^{\ddagger}$ Simon Van Herck, ${ }^{\|}$Bruno G. De Geest, \\ Alain M. Jonas, ${ }^{*}+{ }^{\dagger}$ and Sophie Demoustier-Champagne, ${ }^{*}+{ }^{+}$ \\ ${ }^{\dagger}$ Institute of Condensed Matter and Nanosciences, Université catholique de Louvain, Croix du Sud 1 L7.04.02, B1348. \\ Louvain-la-Neuve, Belgium \\ "Department of Pharmaceutics, Ghent University, Ottergemsesteenweg 460, B9000 Ghent, Belgium
}

\section{Supporting Information}

ABSTRACT: Anisotropic nanostructures, such as nanotubes, incorporating bioactive molecules present interesting features for application as drug delivery carriers. Here, we present the synthesis of layer-by-layer (LbL) nanotubes including protein (ovalbumin) layers and go from simple to more complex synergetic combinations of synthetic and natural polyelectrolytes, leading to structures with tunable properties. The rigidity in organic and aqueous media, the stability in buffer solution and the uptake of different LbL tubes by dendritic cells (DCs) are analyzed to contrast size and chemistry. The most rigid studied systems appear as the best candidates to be internalized by cells, regardless of the chemistry of their outermost layers. The successful transport of long protein-loaded robust rigid nanotubes to the cytoplasm of DCs paves the way for their use as new cargo for the delivery of large amounts of antigen to such cells.

\section{INTRODUCTION}

Micro- and nanosized particles are an interesting alternative to conventional drug carriers due to their potential to minimize dosage, spread it over time, and localized delivery. ${ }^{1}$ Nanometer sized objects are also advantageous to cross biological barriers, whereas the large surface-to-volume ratio inherent to submicron sized objects opens new and exciting possibilities to develop better personalized therapies.

Layer-by-layer (LbL) deposition is a well-established technique with strong potential for the development of drug carriers. $^{2-4}$ Its versatility, simplicity, and use of aqueous conditions makes it an interesting alternative to prepare micro- and nanomaterials of diverse geometry, combining many possible components of complementary charge and chemistry. The method is also attractive because it allows to incorporate a large range of functionalities, some of them possibly helping drugs to reach specific sites and/or to exhibit sustained release.

So far, most research efforts for LbL-based drug delivery have addressed the synthesis of spherical particles, such as proteincontaining nanocapsules or spherical nanoparticles. ${ }^{5-9}$ However, recent reports suggest that the use of an anisotropic geometry of higher aspect ratio would increase the chances of internalization. ${ }^{10}$ Moreover, the shape of the carriers may as well affect the crossing speed of cellular barriers and their performance. ${ }^{11-13}$ Therefore, it appears timely and relevant to study the LbL synthesis of high aspect ratio nanotubes as potential drug carriers.
Nanotubes can be prepared via LbL assembly using a sacrificial hard-template, such as a porous membrane, into the pores of which polycation and polyanion layers are deposited in an alternate fashion. ${ }^{14,15}$ The membranes commonly employed as $\mathrm{LbL}$ templates are made of polycarbonate (PC) or alumina (AAO). Given that template removal is necessary to obtain free nanotubes, the nature of the membrane restricts the nanotube application, as AAO dissolves at either $\mathrm{pH} \leq 4.5$ or $\mathrm{pH} \geq 8.5$, conditions which are not frequently compatible with components of biological origin, whereas PC dissolution requires an organic solvent, such as dichloromethane, conditions which are hardly better. LbL templating must therefore lead to assemblies able to resist the contact with acid, base or organic solvent to successfully recover the nanotubes. For nanotubes used for drug delivery, which include biocompatible elements such as polysaccharides, proteins or polypeptides, this can be challenging. We and several authors have reported the synthesis of protein ${ }^{16-19}$ and hybrid ${ }^{20-23}$ $\mathrm{LbL}$ nanotube architectures for biomedical applications. Though, among those, only one work explored the interaction of free LbL nanotubes with cells. ${ }^{24}$

Among possible applications of protein-based LbL nanotubes, we focus our attention on vaccine vectors to boost the immune response against codelivered antigens. A recently developed strategy is targeting the in situ delivery of an antigen

Received: September 18, 2017

Revised: October 25, 2017

Published: November 13, 2017 
to dendritic cells (DCs), ${ }^{25,26}$ which capture and present it at their surface to T-cells. For example, the ovalbumin protein and its derivate peptide SIINFEKL have been used as model antigens and encapsulated in spherical LbL-particles, ${ }^{26,27}$ thereby proving that $\mathrm{T}$-cell cross presentation in dendritic cells is enhanced in vitro and in vivo with submicron $\mathrm{LbL}$ carriers.

In the present work, we report on the hard-templating combined with LbL deposition of long protein-based polyelectrolyte nanotubes of different chemistry, on their stability in aqueous media and on their in vitro uptake by DCs. We use the term long nanotubes to refer to structures of 400 or $500 \mathrm{~nm}$ diameter having an aspect ratio of about 25 or 40 , respectively. In a first stage, we fabricate $\mathrm{LbL}$ nanotubes based on synthetic polyelectrolytes or on ovalbumin and polypeptides as building blocks. We evaluate their stability in organic solvents and in aqueous media, and then compare their uptake by DCs. Based on these results, we propose a core-shell nanotube architecture consisting of a biocompatible proteinrich compartment and a rigid synthetic compartment, reminiscent of an earlier proposition by Rubner et al., ${ }^{24}$ and study cell internalization for long times of interaction with DC cells and different nanotube architectures (in contrast to Rubner et al.'s previous study ${ }^{24}$ which concentrated on adsorption for much shorter times). Our study demonstrates that the uptake of long, intact nanotube by dendritic cells is possible, provided a proper architecture be selected for the nanotubes.

\section{MATERIALS AND METHODS}

Materials. Poly(allylamine hydrochloride) (PAH, Mw: $58 \mathrm{kDa}$ ), poly(sodium 4-styrenesulfonate) (PSS, $M_{\mathrm{w}}: 70 \mathrm{kDa}$ ), poly-L-arginine (PLA, $M_{\mathrm{w}}>70 \mathrm{kDa}$ ), ovalbumin (OVA, $M_{\mathrm{w}}: 45 \mathrm{kDa}$ ), and poly(allylamine hydrochloride) fluorescein isothiocyanate ( $\mathrm{PAH}^{*}$, $M_{\mathrm{w}}: 15 \mathrm{kDa}$ maximum excitation at $495 \mathrm{~nm}$ and maximum emission at $521 \mathrm{~nm}$ ) with a monomer to dye ratio (PAH/FITC) of (50:1), dichloromethane $\left(\mathrm{CH}_{2} \mathrm{Cl}_{2}\right)$, sodium chloride, $\mathrm{N}$-hydroxysulfosuccinimide sodium salt (sulfo-NHS), $\mathrm{N}$-(3-(dimethylamino)propyl)- $\mathrm{N}^{\prime}$ ethylcarbodiimide hydrochloride (EDC), dextran from Leuconostoc spp., $M_{\mathrm{w}}: 40 \mathrm{kDa}$, and ethylenediaminetetraacetic acid (EDTA) were purchased from Sigma-Aldrich. Poly-L-ornithine hydrobromide (PLO) Mw $78 \mathrm{kDa}$, was acquired from Alamanda Polymers. Sodium hyaluronate (HA, $M_{\mathrm{w}}$ : $176-350 \mathrm{kDa}$ ) was purchased from Life Core. Ovalbumin Alexa Fluor 488 (OVA*) was purchased from Life Technologies. The Alexa Fluor 488 fluorophore has a maximum excitation peak at $496 \mathrm{~nm}$ and a maximum emission peak at $519 \mathrm{~nm}$, according to the supplier. Dulbecco's phosphate-buffered saline (PBS) without calcium nor magnesium was purchased from ThermoScientific. Tris(hydroxymethyl)aminomethane (Tris) was purchased from Acros. (S)-2-Pyridylthio cysteamine hydrochloride (PDA) was bought from CarboSynth Limited. Fluorescein functionalized RGD peptide with a cysteine end (FITC- KRGDSPC) was purchased from Genecust. All aqueous solutions were prepared using deionized water with a resistivity of $18.2 \mathrm{M} \Omega . \mathrm{cm}$ at $25^{\circ} \mathrm{C}$.

Sheets of track-etched PC membranes were provided by It4ip (Louvain-la-Neuve, Belgium, http://www.it4ip.be) with the following dimensions: $400 \mathrm{~nm}$ pore diameter, $10 \mu \mathrm{m}$ thickness and $1 \times 10^{8}$ pores $\cdot \mathrm{cm}^{-2}$, and a different set with $500 \mathrm{~nm}$ pore diameter, $21 \mu \mathrm{m}$ thickness, and $1 \times 10^{8}$ pores $\cdot \mathrm{cm}^{-2}$. Pore diameters of the PC membrane can vary by about 5-10\%. Hydrophilic poly(ethylene terephtalate) (PET) membranes with a pore size of $200 \mathrm{~nm}$ were also provided by It4ip.

All polyelectrolyte solutions were prepared at $1 \mathrm{mg} \cdot \mathrm{mL}^{-1}$ concentration except for fluorescent $\mathrm{OVA}^{*}$ and $\mathrm{PAH}^{*}$, which were prepared at 0.1 and $0.5 \mathrm{mg} \cdot \mathrm{mL}^{-1}$, respectively. Polyelectrolytes were typically dissolved in $150 \mathrm{mM} \mathrm{NaCl}$ without any $\mathrm{pH}$ adjustment, unless stated otherwise.

LbL Assembly by Dipping. The PC membrane was alternately dipped in polycation and polyanion solutions for $30 \mathrm{~min}$, starting with the polycation adsorption since the PC membrane is negatively charged. Each adsorption step was followed by an intermediate twostep rinsing ( $2 \mathrm{~min}$ each) in two baths of aqueous solution of same ionic strength and $\mathrm{pH}$ as used for the polyelectrolytes. Next, the membrane was dipped in the polyanion solution for $30 \mathrm{~min}$ and rinsed (two steps, $2 \mathrm{~min}$ each). The surface of the membrane was cleaned with a cotton swab to prevent pore blockage. The process was repeated until the desired number of layer pairs was obtained. LbLmodified PC membranes were rinsed abundantly with deionized water and subsequently immersed in PBS buffer for at least $24 \mathrm{~h}$ at room temperature.

LbL assembly by filtration. A circular piece of PC membrane was held in a stainless steel syringe filter holder and the polycation/ polyanion solutions were alternately passed through the membrane to adsorb polyelectrolyte layers in the pores of the membrane $(3 \mathrm{~mL}$ of solution for each step, $\left.1 \mathrm{~mL} \cdot \mathrm{min}^{-1}\right)$. Two rinsing steps followed similarly, using solutions of same ionic strength and $\mathrm{pH}$ as the polyelectrolyte ones (twice, $3 \mathrm{~mL}$ of solution for each step, $1 \mathrm{~mL}$. $\left.\mathrm{min}^{-1}\right)$. Immediately after rinsing, the surface of the membrane was cleaned with a cotton swab to prevent pore blockage. Then, a layer of an oppositely charged polyelectrolyte was filtered and the process was repeated until three bilayers of polycation/polyanion. LbL-modified PC membranes were rinsed abundantly with deionized water and immersed in PBS buffer for at least $24 \mathrm{~h}$ at room temperature.

FITC-Peptide Conjugation on LbL-Nanotubes. The protocol followed by Caruso et al. ${ }^{28}$ was adapted to conjugate a cysteine-FITCfunctionalized peptide on HA polymer chains. Briefly, a PC membrane modified by LbL assembly and containing HA layers was immersed overnight in a solution containing $25 \mathrm{mM}$ EDC, $48 \mathrm{mM}$ sulfo-NHS, and $50 \mathrm{mM}$ PDA to install disulfide bonds on the tubes. Then the membrane was extensively rinsed in deionized water and subsequently immersed in a $0.1 \mathrm{mg} / \mathrm{mL}$ FITC-KRGDSPC peptide solution in TrisEDTA buffer $(10 \mathrm{mM}, \mathrm{pH} 7.5)$ for $24 \mathrm{~h}$ to promote FITC conjugation via thiol-disulfide exchange. Finally, the membrane was extensively rinsed in deionized water and stored at $4{ }^{\circ} \mathrm{C}$.

Gas Flow Porometry. Average pore diameter of PC membranes was calculated after gas-flow porometry measurements before and after $\mathrm{LbL}$ assembly. For the measurement, a dry PC membrane was firmly held inside a membrane holder with an effective section area of 0.396 $\mathrm{cm}^{2}$. Nitrogen gas at room temperature was flown upstream with a pressure ranging from 4 to $10 \mathrm{psi}$. The gas flow rate $(\mathrm{mL} / \mathrm{min})$ downstream from the sample was measured by a flowmeter (Agilent). The inner diameter of the pores was calculated by using Knudsen diffusion and the viscous or Hagen-Poiseuille flow relationship, as described elsewhere. ${ }^{29}$

Releasing LbL-Nanotubes from the Template for Imaging. The LbL-modified PC membrane was immersed in dichloromethane and gently stirred to dissolve PC. The nanotubes were then recovered by filtration of the colloidal suspension over a PET membrane. To maximize the removal of remnant PC, fresh $\mathrm{CH}_{2} \mathrm{Cl}_{2}$ was flushed through the PET filters several times.

Collection and Redispersion of LbL-Nanotube in Water or PBS Solutions. We used a previously reported protocol to transfer the LbL nanotubes into an aqueous solution and avoid agglomeration. ${ }^{30}$ Briefly, $20-25 \mathrm{mg}$ of dextran powder was dispersed in a few $\mathrm{mL}$ of dichloromethane and filtered over a PET membrane to form a porous dextran cake. Separately, $1 \mathrm{~cm}^{2}$ of LbL-modified PC membrane was dissolved in an equal volume of dichloromethane and mixed with 20-25 mg of dextran powder before being filtered on top of the dextran cake. An excess of $\mathrm{CH}_{2} \mathrm{Cl}_{2}$ was flushed throughout the porous pellet of nanotubes and dextran to ensure full PC removal. Immediately after the solvent was evaporated, the pellet made of dextran and nanotubes (ca. $1 \times 10^{8}$ nanotubes) was mixed with 1-2 $\mathrm{mL}$ of water or sterile PBS buffer and the suspension was stirred for 15 min, allowing the tubes to be dispersed in the aqueous solution after dextran dissolution. Template removal and nanotube dispersion of the 
Scheme 1. Schematic of the Elaboration of LbL Nanotubes in Polycarbonate (PC) Templates and of $\mathrm{Their}^{\mathrm{Re}} \mathrm{Tease}$ in $\mathrm{CH}_{2} \mathrm{Cl}_{2}$ and Their Dispersion in PBS Buffer

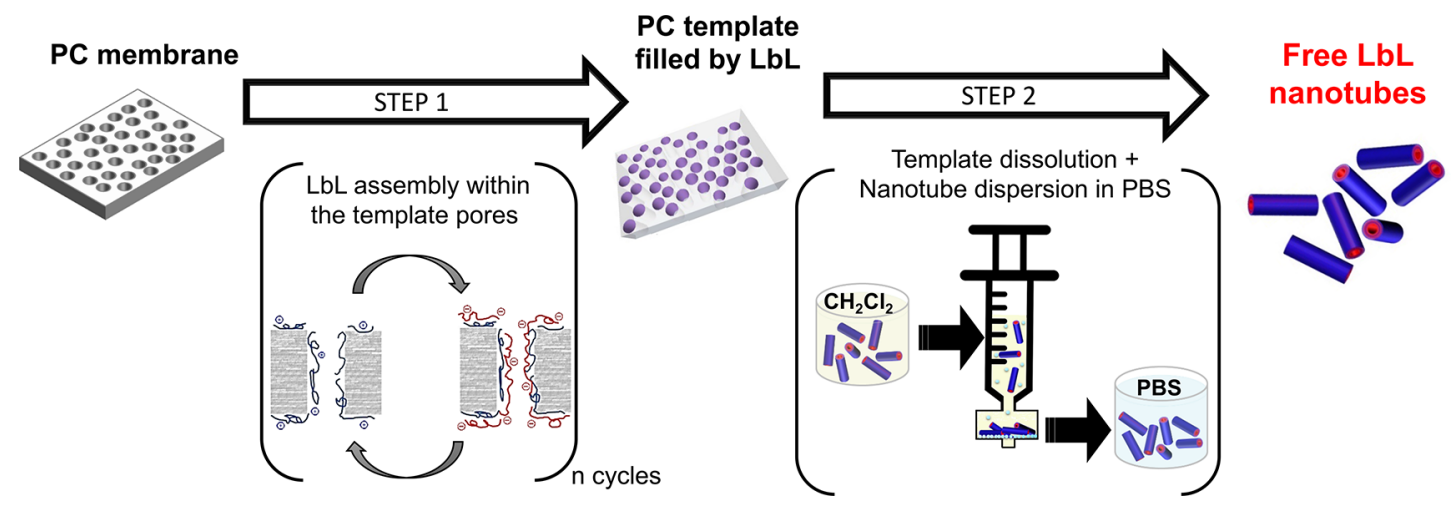

Table 1. Summary of the Composition and Build-up Conditions of the LbL Nanotubes ${ }^{a}$

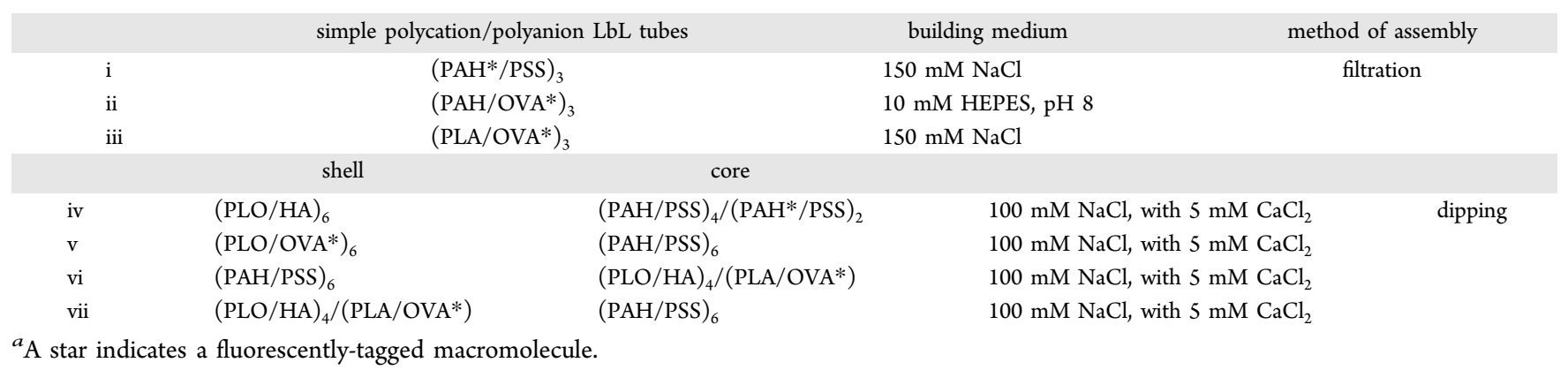

tubes used for cell assays was done in sterile conditions (biologically safe laminar flow hood) to avoid bacterial contamination.

STEM Microscopy. A few droplets of the nanotube dispersion (in PBS or dichloromethane) were dropped over a TEM grid (200 mesh, Electron Microscopy Sciences), followed by a few more droplets of pure water or dichloromethane. Then, the grid was left to dry at room temperature.

The samples were observed at $30 \mathrm{keV}$ with a Field Emission Scanning Electron Microscope (JSM-7600F, Jeol Ltd.) equipped with a transmission detector.

Epi-Fluorescence Microscopy. The suspension of fluorescenttagged nanotubes was either observed dry after collection on a PET filter, or observed in the wet state in a chambered coverglass. Fluorescence images were obtained by an Olympus IX2 inverted microscope equipped with a FITC filter set.

Calculation of LbL Nanotube Rigidity. Epi-fluorescence and STEM images were analyzed using the Image-J software to calculate the rigidity index of the tubes which is defined as $\left\langle R_{\mathrm{EE}}{ }^{2}\right\rangle / \mathrm{L}^{2}$, as we previously reported. ${ }^{30}$ The root-mean-square end-to-end distance of the nanotubes $\left\langle R_{\mathrm{EE}}{ }^{2}\right\rangle^{1 / 2}$ and their average contour length $\langle L\rangle$ were measured for at least 25 different tubes for each system to obtain meaningful average and standard deviation values.

Cell Culture. DC2.4 cells were cultured in DMEM (Dulbecco's Modified Eagle Medium) supplemented with 10\% FBS (fetal bovine serum) and antibiotics (100 units per $\mathrm{mL}$ penicillin and $100 \mu \mathrm{g} \cdot \mathrm{mL}^{-1}$ streptomycin). Cells were grown at $37^{\circ} \mathrm{C}$ in humidified air containing $5 \% \mathrm{CO}_{2}$. DC2.4 cells were plated out on Willco-Dish glass bottom dishes (30000 cells, suspended in $200 \mu \mathrm{L}$ of culture medium) and incubated overnight. Next, the cells were pulsed with $10 \mu \mathrm{L}$ of nanotubes stock solutions and incubated overnight at $37{ }^{\circ} \mathrm{C}$. The following day, cells were fixed and stained with Hoechst and CTBAF555. Briefly, culture medium was aspirated and cells were washed with PBS. Next, $200 \mu \mathrm{L}$ of $4 \%$ paraformaldehyde was added and allowed to fixate for $30 \mathrm{~min}$. A staining solution was prepared by adding blue Hoechst (10 $\mu \mathrm{L}$, of a $2 \mathrm{mg} / \mathrm{mL}$ stock in PBS) and red CTB-AF555 (5 $\mu \mathrm{L}$ of a $1 \mathrm{mg} / \mathrm{mL}$ stock in PBS) to a PBS buffer containing $1 \%$ of BSA $(2 \mathrm{~mL})$. After aspiration and washing, $200 \mu \mathrm{L}$ of this staining solution was added to the fixed cells and incubated for 30 min at $37{ }^{\circ} \mathrm{C}$. Finally, the samples were washed with $1 \%$ BSA PBS buffer and stored on ice.

Confocal Microscopy. Confocal microscopy was carried out with a Leica DMI6000 B inverted microscope equipped with an oil immersion objective (Leica, magnification: 63×, numerical aperture: 1.40) and attached to an Andor DSD2 confocal scanner. Images were processed with ImageJ and Imaris software.

\section{RESULTS AND DISCUSSION}

We assembled polyelectrolyte nanotubes within the pores of polycarbonate membranes via $\mathrm{LbL}$, promoting polyelectrolyte adsorption by filtration or dipping methods (Scheme 1, step 1).

Both methods lead to LbL nanotubes, but we observed that the pores of a membrane are saturated "faster" (with less LbL cycles) by filtration. Our observations indicate that thicker layers can be obtained when the polyelectrolyte solution is forced through the pores by applying pressure (data not shown here). Thus, in a first stage we used filtration to quickly synthesize and analyze three different polyelectrolyte pairs. Yet, to obtain core-shell nanotubes, we used dipping since we target to add at least two different polyelectrolyte pairs with different chemistry. Moreover, the dipping method allows us to produce larger amounts of sample in parallel (contrary to filtration, where the area of the gasket is a limiting factor), which is of relevance for the cell assays.

Different polyelectrolytes pairs were used to compare the properties of the resulting nanotubes, ranging from fully synthetic to protein-based materials, made either from a single pair of polyelectrolytes or comprising compartments of different compositions (hereafter called core-shell structures), as indicated in Table 1.

Simple Polycation/Polyanion LbL Nanotubes. In the first stage, we selected three simple polycation/polyanion 
systems of contrasting chemistry (Table 1, i-iii), that is, (i) $\mathrm{PAH} / \mathrm{PSS}$, as quintessential synthetic robust polyelectrolyte pair; (ii) PAH/OVA to insert a protein as a polyanion while keeping a synthetic polycation, and finally, (iii) PLA/OVA with exclusively polypeptide and protein layers. For these experiments, we used a PC membrane of $500 \mathrm{~nm}$ nominal pore size and $21 \mu \mathrm{m}$ thickness. LbL-modified membranes were dried and then brought in contact with dichloromethane to release the tubes from the template. To evaluate nanotubes stability, we compared them by epi-fluorescence (Figure 1) after their
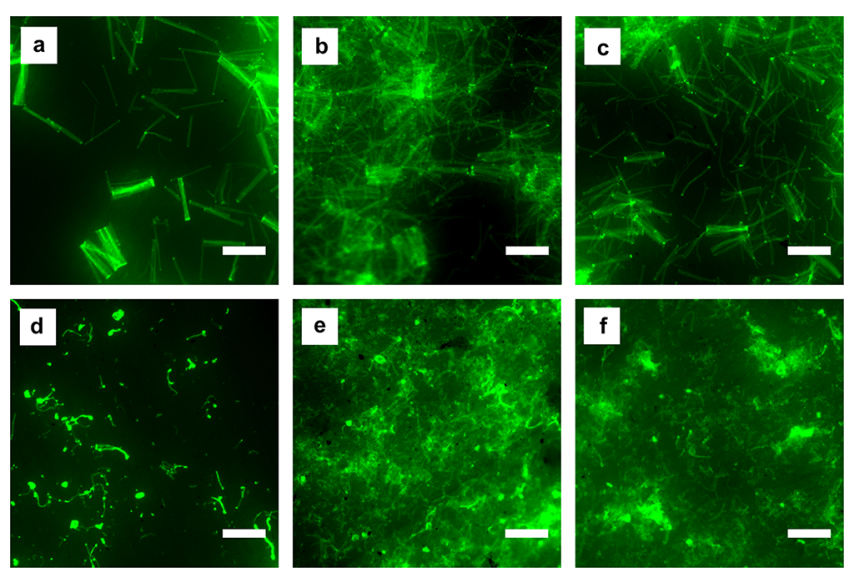

Figure 1. Epi-fluorescence images of LbL nanotubes of different composition in methylene chloride $(\mathrm{a}-\mathrm{c})$ and after $(\mathrm{d}-\mathrm{f})$ immersion in PBS buffer for $24 \mathrm{~h}$; left column $\left(\mathrm{PAH}^{*} / \mathrm{PSS}\right)_{3}(\mathrm{a}, \mathrm{d})$; central column $\left(\mathrm{PAH} / \mathrm{OVA}^{*}\right)_{3}(\mathrm{~b}, \mathrm{e})$; right column $\left(\mathrm{PLA} / \mathrm{OVA}^{*}\right)_{3}(\mathrm{c}, \mathrm{f})$. All tubes are initially $500 \mathrm{~nm}$ in diameter and $21 \mu \mathrm{m}$ in length. Scale bar is $20 \mu \mathrm{m}$.

release in organic solvent $\left(\mathrm{CH}_{2} \mathrm{Cl}_{2}\right.$, sample collected right away) or $24 \mathrm{~h}$ after their transfer into an aqueous solution (PBS buffer), Scheme 1 (step 2). The length of the nanotubes after template removal corresponds to the characteristic thickness of the PC template (i.e., $21 \mu \mathrm{m}$ ), as can be easily measured in the top row of Figure 1, showing tubes collected from the methylene chloride suspension. However, there is a remarkable difference with the second row of images, which shows tubes after a $24 \mathrm{~h}$ dispersion in an aqueous solution, implying that the LbL tubes are at least partially disrupted after being dispersed in water. In addition, whereas PAH/PSS based tubes partially preserve their geometrical features, OVA-containing nanotubes are completely broken into pieces and strongly swollen after exposure to water.

In a former publication, ${ }^{30}$ we introduced a rigidity index to compare the morphology of the nanotubes in a more quantitative manner. The rigidity index, $\left\langle R_{\mathrm{EE}}{ }^{2}\right\rangle / L^{2}$, is defined as the ratio between the root-mean-square of the end-to-end distance $\left(R_{\mathrm{EE}}\right)$, and the square of the average contour length (L). $\left\langle R_{\mathrm{EE}}{ }^{2}\right\rangle / L^{2}$ is a dimensionless parameter ranging from 0 to 1: For a very rigid tube of persistence length $L_{\mathrm{p}} \gg L,\left\langle R_{\mathrm{EE}}^{2}\right\rangle=$ $L^{2}$ and the index of rigidity equals 1 ; for a less rigid tube with $L_{\mathrm{p}}$ $\ll \mathrm{L}$, the index of rigidity is $2 L_{\mathrm{p}} / L<1$, approaching 0 for an infinitely flexible tube of zero persistence length. Using epifluorescence images, we calculated the index of rigidity (Figure 2, bars i-iii). Nanotubes made of simple polyelectrolyte pairs are generally very rigid and of well-preserved shape in the organic solvent as shown by a rigidity index close to 1 . However, the nanotubes lose rigidity after water contact, with the loss being smaller for PAH/PSS and higher for PLA/OVA.

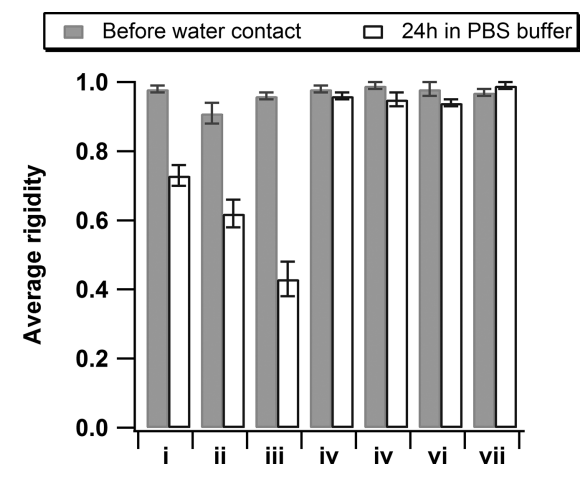

Figure 2. Rigidity index $\left(\left\langle R_{\mathrm{EE}}{ }^{2}\right\rangle / L^{2}\right)$ for all the free polyelectrolyte nanotubes of Table 1 . The error bars represent the standard error on the average value of at least 25 measurements.

This is due to the swelling of the tubes by water, resulting in a decreased Young's modulus, hence decreased persistence lengths and rigidity indices. This result shows that, when dispersed in an aqueous solution, the tubes soften due to swelling, then deform and break more easily for biomacromolecule-based assemblies, a trend in good agreement with a previous report. $^{30}$

The wall thickness of the $\mathrm{LbL}$ tubes of Figure 1 was calculated after gas flow porometry measurements and compared against our observations from Scanning Transmission Electron Microscope (STEM) images. Based on gas flow porometry we calculated the pore size of the membrane before and after LbL deposition, leading to an estimation of the wall thickness of the tubes, $d_{\text {wall }}$ (Table 2). Whereas the

Table 2. Dimensions of the LbL Nanotubes Prepared by Filtration $^{a}$

$\begin{array}{lccc} & \begin{array}{c}\text { nanotubes outer } \\ \text { diameter (from } \\ \text { STEM images) }\end{array} & \begin{array}{c}\text { dry wall thickness } \\ \text { (gas-flow } \\ \text { porometry) }\end{array} & \\ & D_{\text {tube, }}, \mathrm{nm} & D_{\text {wall }}, \mathrm{nm} & D_{\text {tube }} / D_{\text {pore }} \\ (\mathrm{PAH} / \mathrm{PSS})_{3} & 570 \pm 35 & 35 & 1.29 \pm 0.22 \\ (\mathrm{PAH} / \mathrm{OVA})_{3} & 781 \pm 84 & 60 & 1.77 \pm 0.40 \\ (\mathrm{PLA} / \mathrm{OVA})_{3} & 736 \pm 61 & 60 & 1.67 \pm 0.32\end{array}$

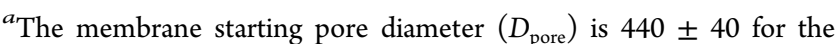
three samples mentioned above.

nominal pore size of the template is $500 \mathrm{~nm}$, we measured about $440 \pm 40 \mathrm{~nm}$ before any polyelectrolyte adsorption. The $(\mathrm{PAH} / \mathrm{PSS})_{3}$ tubes have thinner walls compared to the proteinbased tubes, which is due to the synthetic layers adsorbing in compact "flat" layers. Interestingly, the tubes measured by STEM after template removal in $\mathrm{CH}_{2} \mathrm{Cl}_{2}$ have an external diameter larger than the starting pore size of the template, indicating a "flattening effect" that occurs when the tubes are released from the membrane. The ratio between the diameter of the flattened tubes to the starting diameter of the pores, $D_{\text {tube }} / D_{\text {pore }}$, is an indicator of this flattening and of the softness of the tubes; this ratio is larger for protein-based tubes, which is in good agreement with their lower index of rigidity (Figure 2, i-iii).

For the in vitro uptake of these nanotubes by DCs, they were dispersed in PBS, pulsed on DCs and incubated overnight. Afterward, the culture medium was removed and the cells were fixed and stained for confocal imaging. To gather more information about the uptake and the impact of the nanotube 
size we also included sets of shorter tubes, of 10 versus $21 \mu \mathrm{m}$ tube length. Figure 3 shows the confocal images acquired for
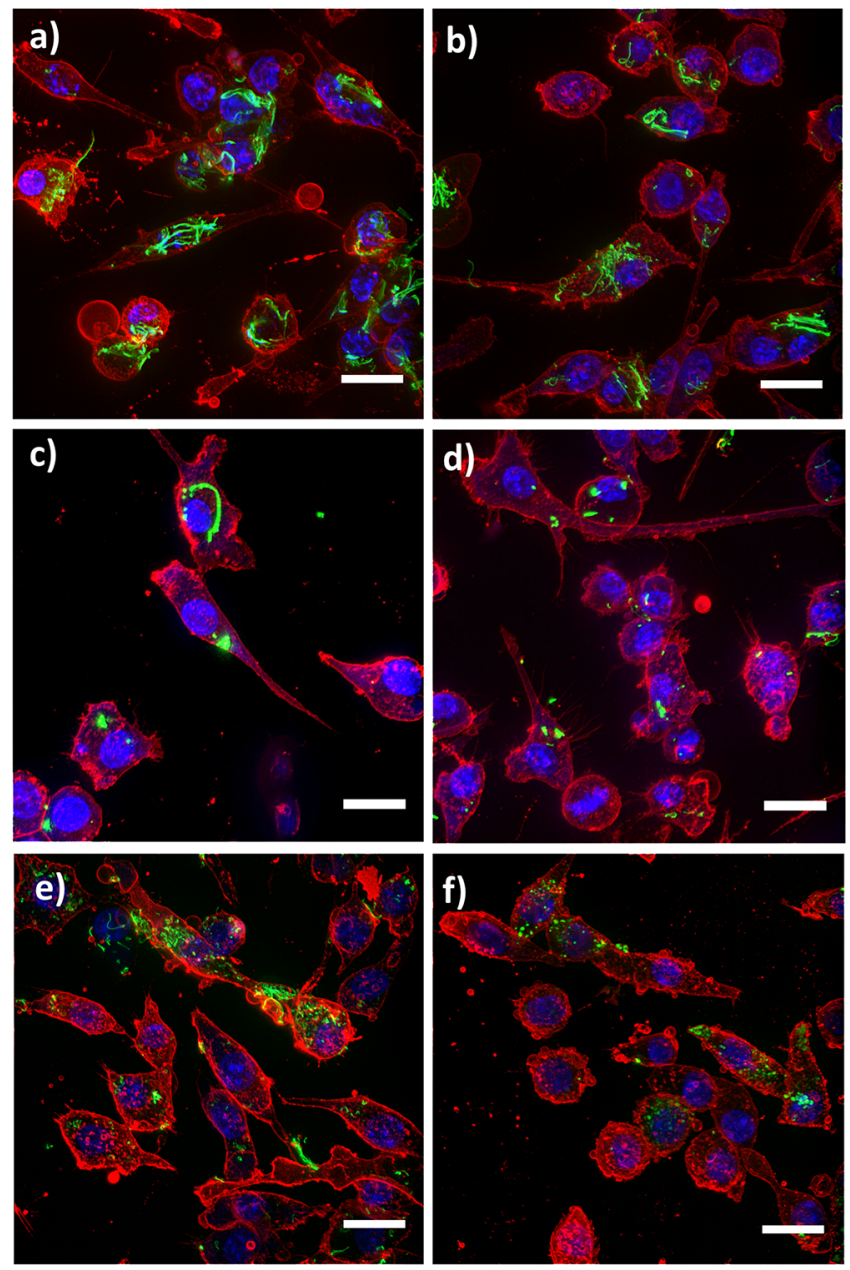

Figure 3. Uptake of LbL nanotubes of different size and chemistry by dendritic cells: top ( $\mathrm{a}, \mathrm{b})$ : $\left(\mathrm{PAH}^{*} / \mathrm{PSS}\right)_{3}$; midsection $(\mathrm{c}, \mathrm{d})$ : (PAH/ OVA $*)_{3}$; bottom $(\mathrm{e}, \mathrm{f})$ : $(\text { PLA/OVA })_{3}$. Left column images $(\mathrm{a}, \mathrm{c}, \mathrm{e})$ are for tubes of $500 \mathrm{~nm}$ starting nominal diameter and $21 \mu \mathrm{m}$ length; the right column (b, d, e) are for tubes of $400 \mathrm{~nm}$ starting nominal diameter and $10 \mu \mathrm{m}$ length The polyelectrolytes indicated with a “*” are stained by FITC (green), while the cell membrane is stained red and the nuclei are stained blue.

the uptake, where we notice that the three types of LbL nanotubes penetrate the cell membrane. Moreover, we found that the cells are viable after overnight exposure to our tubes. Figure 3 reveals that fully synthetic PAH/PSS tubes (Figure $3 a, b)$ preserve their shape and dimensions in the cell (and culture medium). While the Figure $3 \mathrm{~b}$ shows several wellextended tubes, some long (PAH/PSS $)_{3}$ tubes clearly bend to fit inside the cells (DCs have a minor axis of ca. $15 \mu \mathrm{m}$ ). The second row (Figure 3c,d) corresponds to (PAH/OVA) ${ }_{3}$ : the tubes are clearly broken except for a single tube, both the fractured bits and the intact tube being inside the cells. The bottom row (Figure 3e,f) shows that (PLA/OVA) 3 tubes are mostly present as very small fragments inside DCs. The fragmentation of the protein-based nanotubes in the DCs points to their high softness and prohibits concluding on their uptake as tubes by the cells. Clearly, the use of synthetic polyelectrolytes of high linear charge density such as PAH/PSS, which are known to interact strongly, is required to preserve tube shape and study internalization. Preserving tube shape might be important for nanotube internalization, since recent studies have demonstrated that hard particles are more easily phagocytosed compared to soft particles. ${ }^{31}$

Therefore, in order to prepare stable drug carriers incorporating ovalbumin for immunotherapy, we designed multicompartment nanotubes. Thus, we assembled nanotubes integrating a compartment containing protein or polysaccharide layers, and a compartment made of synthetic layers (PAH/ PSS) in order to provide a rigid framework to the nanostructures (Table 1, iv-vii). These nanotubes will be thereafter called core-shell nanotubes.

Core-Shell LbL Nanotubes. Based on our previous confocal observation that shorter tubes do not have to bend to be internalized (Figure 3), we decided to only work with the PC template of $10 \mu \mathrm{m}$ thickness (and $400 \mathrm{~nm}$ nominal pore diameter). Then, in order to better control the assembly of layers of different polyelectrolyte pairs in the pores of the membranes, we moved from filtration assembly to the dipping method, in which the membrane is dipped into the polyelectrolyte solution for $30 \mathrm{~min}$ for adsorption. The polyelectrolyte pair that is first adsorbed also constitutes the most external compartment of the tubes after template removal, that is, the shell compartment. For these core-shell nanotubes, the biocompatible shell compartment was initially made from (1) poly-L-ornithine as polycation and (2) either hyaluronic acid or ovalbumin as polyanion, and the rigidifying core compartment was made from PAH and PSS (Table 1, iv and v). Since PAH and PSS chains have to diffuse into narrower pores, we also used a higher ionic strength with a small amount of divalent ion. ${ }^{17,32}$ Following a similar procedure as before, at the end of the LbL assembly the membrane was air-dried, the polycarbonate dissolved, the nanotubes collected over a filter or transferred and dispersed in an aqueous solution to be observed by epi-fluorescence. Reasoning that our nanotube collection by filtration can favor deformation of the tubes especially after water contact, we used instead a droplet of dispersed tubes in buffer solution and imaged it directly in the epi-fluorescence microscope. The nanotubes dispersed in PBS buffer were also pulsed into DCs and confocal images acquired after overnight contact.

Figure 4 displays the core-shell LbL tube structures before and after water contact, while Figure 2 (bars iv and v) shows the values of calculated rigidity for these core-shell systems. In general, core-shell nanotubes deform and break to a lesser extent compared to simple assemblies, regardless of the chemical nature of the softer shell compartment; in addition, they exhibit negligible differences of rigidity with less than $5 \%$ loss of rigidity index upon dispersion in an aqueous medium. Apparently, the sole addition of the PAH/PSS compartment improves significantly their stability, probably due to a lower hydrophilicity and lesser sensitivity to $\mathrm{pH}$ or ionic strength fluctuations. This results in the dimensions of the tubes corresponding to the thickness of the template, even after uptake by the DCs (Figure 4e,f).

Once proven that the core-shell architecture with soft and rigid compartments is stable, we explored further possibilities to combine the polyelectrolyte pairs in two compartments, and analyze the influence of polyelectrolyte pair placement in the assembly. As before, we selected a PAH/PSS-based rigidifying compartment; the biocompatible compartment was made of a $(\mathrm{PLO} / \mathrm{HA})_{4}$ polysaccharide layer that can be further functionalized or cross-linked via to the carboxylic acid and amine 

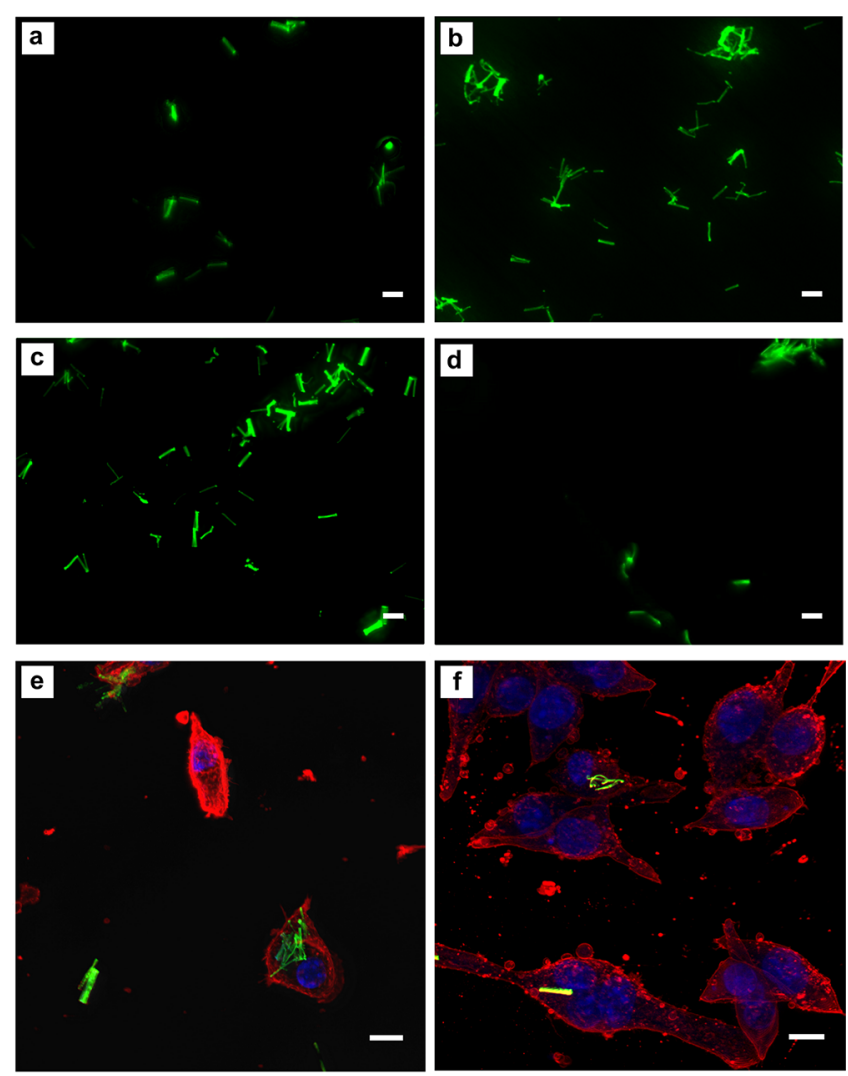

Figure 4. Core-shell LbL nanotubes and their uptake by DCs: (a, c, e) $\left[\right.$ shell of $(\mathrm{PLO} / \mathrm{HA})_{6}$ and core of $\left.(\mathrm{PAH} / \mathrm{PSS})_{4} /\left(\mathrm{PAH}^{*} / \mathrm{PSS}\right)_{2}\right]$; (b, $\mathrm{d}, \mathrm{f})$ shell of $\left(\mathrm{PLO} / \mathrm{OVA}^{*}\right)_{6}$ and core of $(\mathrm{PAH} / \mathrm{PSS})_{6}$. The top images $(\mathrm{a}, \mathrm{b})$ are nanotubes after dissolution of the PC membrane in methylene chloride, whereas the middle row (c, d) are nanotubes suspended in PBS buffer. The amount of tubes is lower than in Figure 3 because the suspension of the tubes in water is directly imaged here. The tubes contain a green fluorescent compartment, the cell membrane is stained red, and the nuclei are stained blue. Scale bars are $10 \mu \mathrm{m}$.

lateral groups with a final PLA/OVA layer for OVA delivery (Table 1, vi and vii). In order to check the effect of the nature of the shell, two series of tubes were made, one in which the rigid compartment is in the shell (vi) and one in which it is in the core (vii). For the uptake studies, we also added one more factor by dispersing the tubes in either (1) PBS or (2) ovalbumin in PBS, in which case we surmised that the very external layer of the tubes would be changed to OVA due to its adsorption on the tubes. The tubes were then immediately placed in contact with DCs.

Figure 5 compares the uptake of $\mathrm{LbL}$ tubes with the rigid synthetic compartment in the shell (a: shell of (PAH/PSS) and core of $\left.(\mathrm{PLO} / \mathrm{HA})_{4} / \mathrm{PLA} / \mathrm{OVA}^{*}\right)$, or in the inner core (c: shell of $(\mathrm{PLO} / \mathrm{HA})_{4} / \mathrm{PLA} / \mathrm{OVA}^{*}$ and core of $\left.(\mathrm{PAH} / \mathrm{PSS})_{6}\right)$. The uptake of the tubes covered by an extra-layer of ovalbumin is presented in Figure $5 \mathrm{~b}$ and d, respectively. All the core-shell tubes show a well-preserved morphology, and their shape and dimensions is kept in aqueous solutions for about 3 months (Supporting Information, SI Figure 1). The rigidity calculations (Figure 2, vi and vii) also reveal that the core-shell tubes are invariably rigid in both organic and aqueous solutions, demonstrating the generality of our two-compartment strategy. Moreover, the confocal images in Figure 5 show no major difference in $24 \mathrm{~h}$ cell uptake for these four samples, as all of
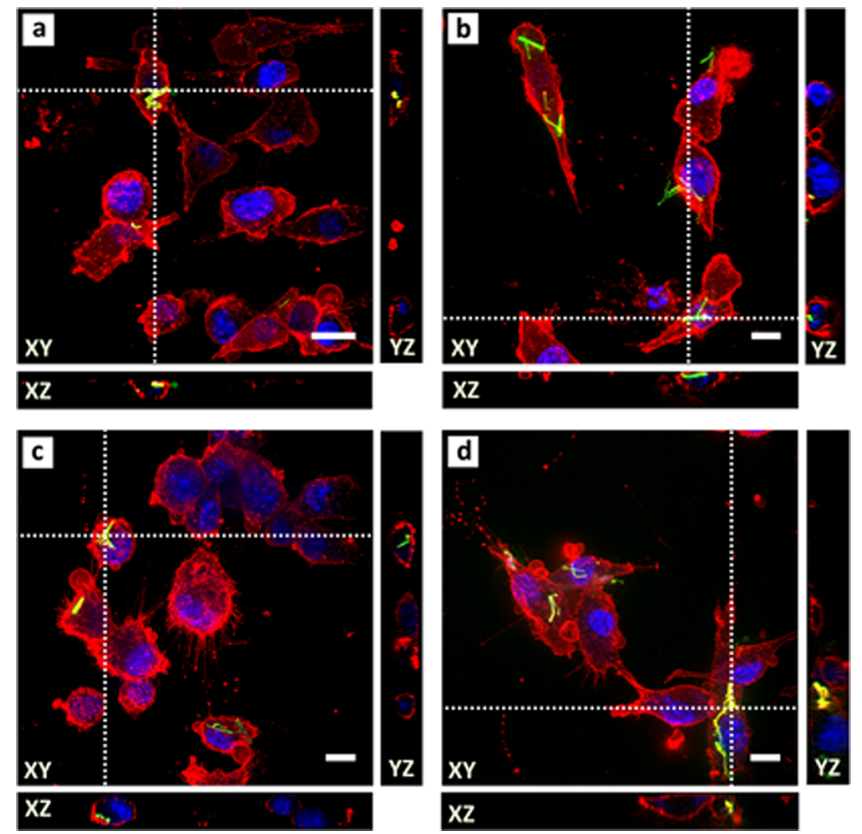

Figure 5. In vitro uptake of $\mathrm{LbL}$ core-shell nanotubes by DCs observed by confocal microscopy, (a) shell of (PAH/PSS) ${ }_{6}$ and core of $(\mathrm{PLO} / \mathrm{HA})_{4} / \mathrm{PLA} / \mathrm{OVA}^{*}$; (b) shell of OVA-(PAH/PSS $)_{6}$ and core of $(\mathrm{PLO} / \mathrm{HA})_{4} / \mathrm{PLA} / \mathrm{OVA}^{*}$; (c) shell of $(\mathrm{PLO} / \mathrm{HA})_{4} / \mathrm{PLA} / \mathrm{OVA}^{*}$ and core of $\left.(\mathrm{PAH} / \mathrm{PSS})_{6} ; \mathrm{d}\right)$ shell of OVA/(PLO/HA $)_{4} / \mathrm{PLA} / \mathrm{OVA}^{*}$ and core of $(\mathrm{PAH} / \mathrm{PSS})_{6}$. Scale bars are $10 \mu \mathrm{m}$. Dotted lines indicate the position of the transversal $X Z$ and $Y Z$ planes.

them penetrate the membrane wall (the projections $X Z$ and $Y Z$ illustrate). In contrast with the results of Rubner et al. on B cells; $^{24}$ this is most probably due to the shorter interaction times used by these authors $(2 \mathrm{~h})$, as well as the different cell nature. At any rate, our results show that whatever the chemical nature of the outer shell, intact rigid tubes as long as $10 \mu \mathrm{m}$ are taken by the dendritic cells, leading to substantial cargo delivery.

\section{CONCLUSIONS}

We demonstrated that robust LbL tubes from 10 to $20 \mu \mathrm{m}$ long can be internalized by DCs while preserving their morphology. The main advantage of long LbL nanotubes as drug carriers is in the amount of active biomolecules contained in a single carrier. For instance, a tube of $10 \mu \mathrm{m}$ length and $500 \mathrm{~nm}$ diameter has a volume that equals about $30 \times$ the one of a spherical particle of $500 \mathrm{~nm}$ diameter. Thus, once internalized, it will deliver a significantly increased loading to the targeted cell.

In addition, long LbL nanotubes are easily distinguishable units, easier to track by microscopy techniques, which is of interest for in vitro studies. It is important to point out that the cellular uptake of the long polyelectrolyte nanotubes presented here raises questions about the internalization pathway. It has been described in the literature that large particles are internalized via macropinocytosis or phagocytosis, ${ }^{8,33}$ similarly as it occurs for viruses and bacteria, with membrane channels as large as $5 \mu \mathrm{m} .{ }^{34,35}$ Tracking the endocytosis process by imaging at shorter time intervals until the uptake is complete would be very interesting in this respect.

A careful selection of polyelectrolyte pairs arranged in a core-shell structure is key to obtain stable nanostructures in aqueous dispersions, such as a $\mathrm{PAH} / \mathrm{PSS}$-based rigid compart- 
ment combined with a biocompatible PLO/OVA- and/or PLO/HA-based soft compartment. Contrariwise, LbL tubes that are exclusively based on polysaccharide or protein layers are likely to deform, break, and entangle in aqueous solutions and, therefore, present limited interest. The exact chemical nature of the outer compartment does not affect internalization, with reciprocal core-shell structures behaving identically. More quantitative studies are however required to fully evaluate the efficiency of transfection, which could vary depending on nanotubes architecture.

Overall, our report corroborates the applicability of $\mathrm{LbL}$ assembly and templating methods to build sturdy structures with tunable properties, using a proper combination of natural and synthetic polyelectrolytes acting in synergy. We have also shown how a protein load incorporated in robust rigid tubes could be transported to the cytoplasm of dendritic cells, which offers a new route for the delivery of large amounts of antigen to such cells.

\section{ASSOCIATED CONTENT}

\section{S Supporting Information}

The Supporting Information is available free of charge on the ACS Publications website at DOI: 10.1021/acs.biomac.7b01353.

Stability of the core-shell nanotubes with three compartments followed by Epi-Fluorescence for up to three months (PDF).

\section{AUTHOR INFORMATION}

\section{Corresponding Authors}

*E-mail: sophie.demoustier@uclouvain.be.

*E-mail: alain.jonas@uclouvain.be.

\section{ORCID}

Bruno G. De Geest: 0000-0001-9826-6170

Alain M. Jonas: 0000-0002-4083-0688

Sophie Demoustier-Champagne: 0000-0003-3045-6060

\section{Present Address}

${ }^{\S}$ The Khademhosseini Lab, Harvard-MIT Division of Health Sciences and Technology, 65 Landsdowne Street, Cambridge, MA 02139, U.S.A.

\section{Author Contributions}

${ }^{\ddagger}$ These authors contributed equally. The manuscript was written through contributions of all authors. All authors have given approval to the final version of the manuscript.

\section{Notes}

The authors declare no competing financial interest.

\section{ACKNOWLEDGMENTS}

We thank, it4ip for the track-etched membranes and Karine Glinel for the gift of PLO and HA. Financial support was provided by the Belgian Science Policy (IAP P7/05) and the Belgian Fund for Scientific Research (FNRS).

\section{ABBREVIATIONS}

LbL, layer-by-layer; DC, dendritic cells; PC, polycarbonate; PBS, phosphate buffered saline; $\mathrm{PAH}$, poly(allylamine hydrochloride); PSS, poly(sodium 4-styrenesulfonate); PLA, poly-Larginine; OVA, ovalbumin; PLO, poly-L-ornithine hydrobromide; PBS, phosphate-buffered saline; Tris, Tris(hydroxymethyl)aminomethane

\section{REFERENCES}

(1) Blanco, E.; Shen, H.; Ferrari, M. Principles of nanoparticle design for overcoming biological barriers to drug delivery. Nat. Biotechnol. 2015, 33 (9), 941-51.

(2) Correa, S.; Dreaden, E. C.; Gu, L.; Hammond, P. T. Engineering nanolayered particles for modular drug delivery. J. Controlled Release 2016, 240, 364-386.

(3) Costa, R. R.; Alatorre-Meda, M.; Mano, J. F. Drug nanoreservoirs synthesized using layer-by-layer technologies. Biotechnol. Adv. 2015, 33 (6), 1310-1326.

(4) Guzmán, E.; Mateos-Maroto, A.; Ruano, M.; Ortega, F.; Rubio, R. G. Layer-by-Layer polyelectrolyte assemblies for encapsulation and release of active compounds. Adv. Colloid Interface Sci. 2017.

(5) Delcea, M.; Mohwald, H.; Skirtach, A. G. Stimuli-responsive LbL capsules and nanoshells for drug delivery. Adv. Drug Delivery Rev. 2011, 63 (9), 730-47.

(6) De Koker, S.; Hoogenboom, R.; De Geest, B. G. Polymeric multilayer capsules for drug delivery. Chem. Soc. Rev. 2012, 41 (7), 2867-84.

(7) Cui, J.; van Koeverden, M. P.; Müllner, M.; Kempe, K.; Caruso, F. Emerging methods for the fabrication of polymer capsules. Adv. Colloid Interface Sci. 2014, 207, 14-31.

(8) De Koker, S.; De Cock, L. J.; Rivera-Gil, P.; Parak, W. J.; Auzely Velty, R; Vervaet, C.; Remon, J. P.; Grooten, J.; De Geest, B. G. Polymeric multilayer capsules delivering biotherapeutics. Adv. Drug Delivery Rev. 2011, 63 (9), 748-61.

(9) De Cock, L. J.; De Koker, S.; De Geest, B. G.; Grooten, J.; Vervaet, C.; Remon, J. P.; Sukhorukov, G. B.; Antipina, M. N. Polymeric multilayer capsules in drug delivery. Angew. Chem., Int. Ed. 2010, 49 (39), 6954-73.

(10) He, Y.; Park, K. Effects of the Microparticle Shape on Cellular Uptake. Mol. Pharmaceutics 2016, 13 (7), 2164-71.

(11) Hinde, E.; Thammasiraphop, K.; Duong, H. T.; Yeow, J.; Karagoz, B.; Boyer, C.; Gooding, J. J.; Gaus, K. Pair correlation microscopy reveals the role of nanoparticle shape in intracellular transport and site of drug release. Nat. Nanotechnol. 2016, 12 (1), 8189.

(12) Karagoz, B.; Esser, L.; Duong, H. T.; Basuki, J. S.; Boyer, C.; Davis, T. P. Polymerization-Induced Self-Assembly (PISA) - control over the morphology of nanoparticles for drug delivery applications. Polym. Chem. 2014, 5 (2), 350-355.

(13) Adnan, N. N. M.; Cheng, Y. Y.; Ong, N. M. N.; Kamaruddin, T. T.; Rozlan, E.; Schmidt, T. W.; Duong, H. T. T.; Boyer, C. Effect of gold nanoparticle shapes for phototherapy and drug delivery. Polym. Chem. 2016, 7 (16), 2888-2903.

(14) Liang, Z.; Susha, A. S.; Yu, A.; Caruso, F. Nanotubes Prepared by Layer-by-Layer Coating of Porous Membrane Templates. Adv. Mater. 2003, 15 (21), 1849-1853.

(15) Ai, S.; Lu, G.; He, Q.; Li, J. Highly flexible polyelectrolyte nanotubes. J. Am. Chem. Soc. 2003, 125 (37), 11140-1.

(16) Komatsu, T. Protein-based nanotubes for biomedical applications. Nanoscale 2012, 4 (6), 1910-8.

(17) Zhang, S.; Demoustier-Champagne, S.; Jonas, A. M. Quantitative Collection and Enzymatic Activity of Glucose Oxidase Nanotubes Fabricated by Templated Layer-by-Layer Assembly. Biomacromolecules 2015, 16 (8), 2382-93.

(18) Kalaskar, D. M.; Poleunis, C.; Dupont-Gillain, C.; DemoustierChampagne, S. Elaboration of nanostructured biointerfaces with tunable degree of coverage by protein nanotubes using electrophoretic deposition. Biomacromolecules 2011, 12 (11), 4104-11.

(19) Landoulsi, J.; Roy, C. J.; Dupont-Gillain, C.; DemoustierChampagne, S. Synthesis of collagen nanotubes with highly regular dimensions through membrane-templated layer-by-layer assembly. Biomacromolecules 2009, 10 (5), 1021-4.

(20) Dougherty, S. A.; Zhang, D.; Liang, J. Fabrication of protein nanotubes using template-assisted electrostatic layer-by-layer methods. Langmuir 2009, 25 (22), 13232-7. 
(21) Goto, S.; Amano, Y.; Akiyama, M.; Bottcher, C.; Komatsu, T. Gold nanoparticle inclusion into protein nanotube as a layered wall component. Langmuir 2013, 29 (46), 14293-300.

(22) Roy, C. J.; Chorine, N.; De Geest, B. G.; De Smedt, S.; Jonas, A. M.; Demoustier-Champagne, S. Highly Versatile Approach for Preparing Functional Hybrid Multisegmented Nanotubes and Nanowires. Chem. Mater. 2012, 24 (9), 1562-1567.

(23) Kobayakawa, S.; Nakai, Y.; Akiyama, M.; Komatsu, T. SelfPropelled Soft Protein Microtubes with a Pt Nanoparticle InteriorSurface. Chem. - Eur. J. 2017, 23 (21), 5044-5050.

(24) Gilbert, J. B.; O’Brien, J. S.; Suresh, H. S.; Cohen, R. E.; Rubner, $\mathrm{M}$. F. Orientation-specific attachment of polymeric microtubes on cell surfaces. Adv. Mater. 2013, 25 (41), 5948-52.

(25) Macri, C.; Dumont, C.; Johnston, A. P.; Mintern, J. D. Targeting dendritic cells: a promising strategy to improve vaccine effectiveness. Clin. Transl. Immunol. 2016, 5 (3), e66.

(26) De Koker, S.; Fierens, K.; Dierendonck, M.; De Rycke, R.; Lambrecht, B. N.; Grooten, J.; Remon, J. P.; De Geest, B. G. Nanoporous polyelectrolyte vaccine microcarriers. A formulation platform for enhancing humoral and cellular immune responses. $J$. Controlled Release 2014, 195, 99-109.

(27) De Geest, B. G.; Willart, M. A.; Lambrecht, B. N.; Pollard, C.; Vervaet, C.; Remon, J. P.; Grooten, J.; De Koker, S. SurfaceEngineered Polyelectrolyte Multilayer Capsules: Synthetic Vaccines Mimicking Microbial Structure and Function. Angew. Chem., Int. Ed. 2012, 51 (16), 3862-6.

(28) Chong, S. F.; Sexton, A.; De Rose, R.; Kent, S. J.; Zelikin, A. N.; Caruso, F. A paradigm for peptide vaccine delivery using viral epitopes encapsulated in degradable polymer hydrogel capsules. Biomaterials 2009, 30 (28), 5178-86.

(29) Roy, C. J.; Dupont-Gillain, C.; Demoustier-Champagne, S.; Jonas, A. M.; Landoulsi, J. Growth mechanism of confined polyelectrolyte multilayers in nanoporous templates. Langmuir 2010, 26 (5), 3350-5.

(30) Saghazadeh, S.; Zhang, S.; Lefevre, D.; Le Beulze, A.; Jonas, A. M.; Demoustier-Champagne, S. Universal Method to Transfer Membrane-Templated Nano-Objects to Aqueous Solutions. Langmuir 2015, 31 (26), 7264-73.

(31) Anselmo, A. C.; Zhang, M.; Kumar, S.; Vogus, D. R.; Menegatti, S.; Helgeson, M. E.; Mitragotri, S. Elasticity of nanoparticles influences their blood circulation, phagocytosis, endocytosis, and targeting. ACS Nano 2015, 9 (3), 3169-77.

(32) Rajesh, S.; Yan, Y.; Chang, H. C.; Gao, H.; Phillip, W. A. Mixed mosaic membranes prepared by layer-by-layer assembly for ionic separations. ACS Nano 2014, 8 (12), 12338-45.

(33) Salatin, S.; Maleki Dizaj, S.; Yari Khosroushahi, A. Effect of the surface modification, size, and shape on cellular uptake of nanoparticles. Cell Biol. Int. 2015, 39 (8), 881-90.

(34) Lim, J. P.; Gleeson, P. A. Macropinocytosis: an endocytic pathway for internalising large gulps. Immunol. Cell Biol. 2011, 89 (8), 836-843.

(35) Champion, J. A.; Walker, A.; Mitragotri, S. Role of Particle Size in Phagocytosis of Polymeric Microspheres. Pharm. Res. 2008, 25 (8), $1815-1821$. 